\title{
Doppler tomography of the black hole binary A0620-00 and the origin of chromospheric emission in quiescent X-ray binaries ${ }^{\star}$
}

\author{
J. I. González Hernández ${ }^{1}$ and J. Casares ${ }^{2}$ \\ 1 Dpto. de Astrofísica y Ciencias de la Atmósfera, Facultad de Ciencias Físicas, Universidad Complutense de Madrid, \\ 28040 Madrid, Spain \\ e-mail: jonay@astrax.fis.ucm.es \\ 2 Instituto de Astrofísica de Canarias, C/ Via Láctea s/n, 38200 La Laguna, Spain \\ e-mail: jorge.casares@iac.es
}

Received 18 January 2010 / Accepted 28 March 2010

ABSTRACT

\begin{abstract}
Context. Doppler tomography of emission line profiles in low-mass X-ray binaries allows us to disentangle the different emission sites and study the structure and variability of accretion disks.

Aims. We present UVES high-resolution spectroscopic observations of the black hole binary A0620-00 at quiescence.

Methods. These spectroscopic data constrain the orbital parameters $P_{\text {orb }}=0.32301405(1) \mathrm{d}$ and $K_{2}=437.1 \pm 2.0 \mathrm{~km} \mathrm{~s}^{-1}$. These values, together with the mass ratio $q=M_{2} / M_{1}=0.062 \pm 0.010$, imply a minimum mass for the compact object of $M_{1} \sin ^{3} i=$ $3.15 \pm 0.10 M_{\odot}$, consistent with previous works.

Results. The $\mathrm{H} \alpha$ emission from the accretion disk is much weaker than in previous studies, possibly because of a decrease in disk activity. Doppler imaging of the $\mathrm{H} \alpha$ line shows for the first time a narrow component coming from the secondary star, with an observed equivalent width of $1.4 \pm 0.3 \AA$, perhaps associated to chromospheric activity. Subtracting a K-type template star and correcting for the veiling of the accretion disk yields an equivalent width of $2.8 \pm 0.3 \AA$. A bright hot spot is also detected at the position where the gas stream trajectory intercepts the accretion disk.

Conclusions. The H $\alpha$ flux associated to the secondary star is too large to be powered by X-ray irradiation. It is comparable to those observed in RS CVn binaries with similar orbital periods and is probably triggered by the rapid stellar rotation.
\end{abstract}

Key words. black hole physics - accretion, accretion disks - binaries: close - stars: individual: V616 Mon - X-rays: binaries stars: activity

\section{Introduction}

The black hole binary A0620-00 (V616 Mon) is one of the most studied low-mass X-ray binaries (LMXBs) and is considered as the prototype soft X-ray transient (SXT). It was discovered in 1975 by the Ariel 5 (Elvis et al. 1975) satellite during an X-ray outburst where the optical brightness of the system increased by roughly 6 mag in a few days. One year and several months later the system returned to its quiescent state at $m_{\mathrm{V}} \sim 18.35 \mathrm{mag}$. The spectrum of a stellar counterpart was then identified and classified as a K5V-K7V star (Oke 1977; Murdin et al. 1980). Later spectroscopic observations allowed determination of the orbital period of the secondary star at $\sim 7.75 \mathrm{~h}$ (McClintock \& Remillard 1986), which implied the presence of a black hole of minimun mass $\sim 3.1 M_{\odot}$ (Marsh et al. 1994).

The orbital inclination of the system was later estimated from IR light curves at $\sim 41^{\circ}$, implying a primary black hole mass of $11.0 \pm 1.9 M_{\odot}$ (Gelino et al. 2001; see also Shahbaz et al. 1994). However, this study adopts a K4V stellar component with $T_{\text {eff }} \sim 4600 \mathrm{~K}, 300 \mathrm{~K}$ cooler than the effective temperature $T_{\text {eff }}=$ $4900 \pm 100 \mathrm{~K}$ derived by González Hernández et al. (2004) from high-resolution optical spectroscopic observations. This

* Based on observations obtained with UVES at VLT Kueyen $8.2 \mathrm{~m}$ telescope in programme 66.D-0157(A). may affect the required veiling in the IR, hence the derived inclination and black hole mass (Hynes et al. 2005). Furthermore, Cantrell et al. (2010) present evidence of substantial disk contamination in their IR light curves and find $i=51 \pm 1^{\circ}$, which translates into a lower black hole mass of $6.6 \pm 0.3 M_{\odot}$. On the other hand, contradictory results have been reported using lowresolution IR spectra. Harrison et al. (2007) find a very small or negligible disk veiling at IR wavelengths, whereas Froning et al. (2007) conclude that it can be $18 \%$ in the $\mathrm{H}$ band. The latter also argue that $T_{\text {eff }} \lesssim 4600 \mathrm{~K}$ is needed to fit their observations; however, we must note here that most studies just adopt a $T_{\text {eff }}$ based on spectral classification, derived through comparison with low-resolution template spectra, without determining the true $T_{\text {eff }}$ of the template (Hynes et al. 2005). A similar inconsistency has been found for the black hole binary XTE J1118+480 for which Gelino et al. (2006) adopted a $T_{\text {eff }}=4250 \mathrm{~K}$ where González Hernández et al. (2006, 2008b) derive a spectroscopic $T_{\text {eff }}$ of $4700 \pm 100 \mathrm{~K}$.

The accretion disk of A0620-00 has been studied in the UV (McClintock et al. 1995) and the optical (Marsh et al. 1994; Orosz et al. 1994), allowing investigation of the inner and outer disk. Both works in the UV and optical seem to agree that the accretion disk is in a true quiescent state; however, this does not mean that the disk is inactive and probably its variability 
appears to be relevant (Shahbaz et al. 2004; Cantrell et al. 2008, 2010). Shahbaz et al. (2004) also suggest that the accretion disk in A0620-00 could be eccentric which may have been confirmed by more recent observations reported by Neilsen et al. (2008). As pointed out by these authors, to determine the definite mass of the compact object, it is very important to understand the structure and variability of the accretion disk as exemplified by Cantrell et al. (2010).

Here we present high-resolution spectroscopy of A0620-00 where we detect clear emission arising from the secondary star in the $\mathrm{H} \alpha$ Doppler map. This feature has only been observed before in the systems GU Mus (Casares et al. 1997), Nova Oph 77 (Harlaftis et al. 1997), Cen X-4 (Torres et al. 2002; D’Avanzo et al. 2005) and Nova Scorpii 1994 (Shahbaz et al. 1999). These data also allow us to revisit the orbital parameters of the system, which we find to be consistent with previous studies.

\section{Observations}

We obtained 20 spectra of A0620-00 with the UV-Visual Echelle Spectrograph (UVES) at the European Southern Observatory (ESO), Observatorio Cerro Paranal (Chile), using the $8.2 \mathrm{~m}$ Very Large Telescope (VLT) on 5, 17, and 21 December 2000, covering the spectral regions $\lambda \lambda 4800-5800 \AA$, and $\lambda \lambda 5800$ $6800 \AA$ at resolving power $\lambda / \delta \lambda \sim 43000$, with a dispersion of 0.029 and $0.035 \AA$ pixel $^{-1}$ for the blue and red arms, respectively. The total exposure time was $2.9 \mathrm{~h}$. The spectra were reduced in a standard manner using the UVES reduction package within the MIDAS environment. The exposure time was fixed at $\sim 500 \mathrm{~s}$ to minimize the effects of orbital smearing which, for the orbital parameters of A0620-00, is in the range $2-54 \mathrm{~km} \mathrm{~s}^{-1}$, so in some cases larger than the instrumental resolution of $\sim 7 \mathrm{~km} \mathrm{~s}^{-1}$. The signal-to-noise ratio per pixel in the individual spectra is $\sim 4$ and 8 in continuum regions close to the $\mathrm{H} \beta$ and $\mathrm{H} \alpha$ lines, respectively. Thus the spectra were binned in wavelength with steps of $0.1 \AA$ pixel $^{-1}$, increasing the $S / N \sim 6$ and 12 at $\mathrm{H} \beta$ and $\mathrm{H} \alpha$, respectively. In this paper, we also use the K3-K4.5V template star HD 209100, observed on 12 November 2000 with the Coralie spectrograph, installed on the $1.2 \mathrm{~m}$ Euler Swiss Telescope at the ESO La Silla Observatory (Chile), with a spectral resolution of $\lambda / \delta \lambda \sim 50000$. This spectrum was rebinned to the same step and degraded to the same resolution of the spectra of A0620-00.

\subsection{Revised orbital parameters}

We extracted the radial velocities by cross-correlating each UVES spectrum of the target with the K3-4.5V template spectrum of HD209100, using the software MOLLY developed by Marsh. Here we only concentrated on the $\mathrm{H} \beta$ spectra, which cover the spectral regions $\lambda \lambda 4800-5800 \AA$, because they contain a larger number of metallic absorption lines for the crosscorrelation so provide smaller errorbars by a factor of 2 . In any case, the result of cross-correlating the $\mathrm{H} \alpha$ spectra yields identical results. Prior to the cross-correlation, the template spectrum was rotationally broadened by $96 \mathrm{~km} \mathrm{~s}^{-1}$ to match the rotational velocity of the donor star (see Sect. 2.2).

A $\chi^{2}$ sine wave fit, $V=K_{2} \sin \left[2 \pi\left(t-T_{0}\right) / P\right]$, to the obtained velocities yields the following orbital solution (see Fig. 1): $\gamma=8.5 \pm 1.8 \mathrm{~km} \mathrm{~s}^{-1}, K_{2}=437.1 \pm 2.0 \mathrm{~km} \mathrm{~s}^{-1}$, and $T_{0}=2451883.0313 \pm 0.0002 \mathrm{~d}$, where $T_{0}$ is defined as the heliocentric time of the inferior conjunction of the companion star. The orbital period was initially set to the value reported

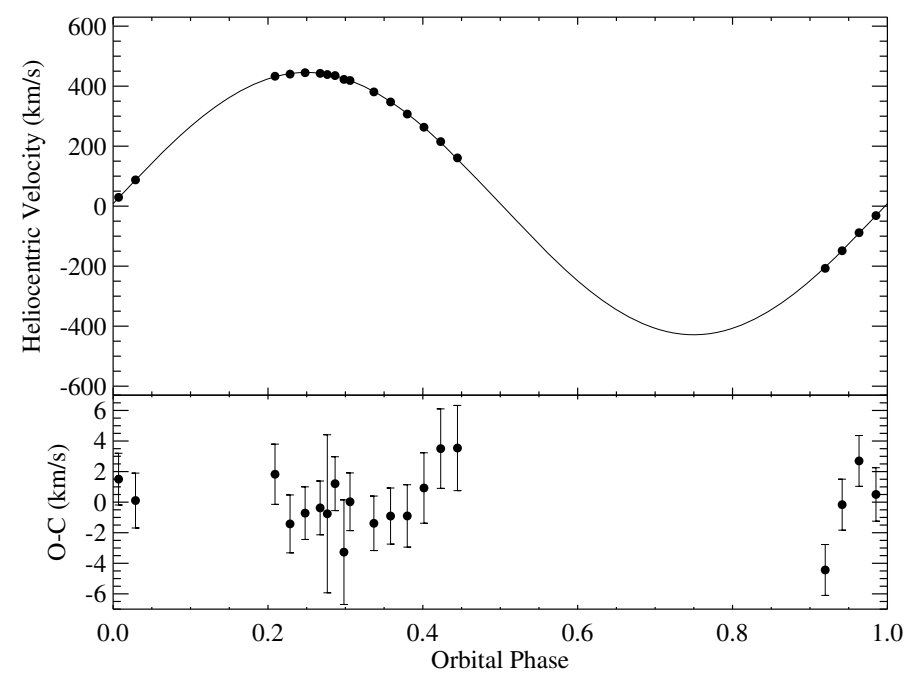

Fig. 1. Top panel: radial velocities of the secondary star in A0620-00 folded on the orbital solution of the data with best fitting sinusoid. Individual velocity errors are $\lesssim 3 \mathrm{~km} \mathrm{~s}^{-1}$ and are not plotted because they are always smaller than the symbol size. Bottom panel: residuals of the fit together with the individual errors that have been rescaled by a factor 1.4 so that the minimum reduced $\chi^{2}$ is 1.0 .

by McClintock \& Remillard (1986) and subsequently refined to $P_{\text {orb }}=0.32301405 \pm 0.00000001 \mathrm{~d}$ after dividing the difference between our $T_{0}$ and the one quoted by Orosz et al. (1994) by an integer number of cycles (17957). The quoted uncertainties are at $1 \sigma$, and we have rescaled the errors by a factor 1.4 so that the minimum reduced $\chi^{2}$ is 1.0 .

This orbital period, $P$, together with the velocity amplitude of the orbital motion of the secondary star, $K_{2}$, leads to a mass function of $f(M)=P K_{2}^{3} / 2 \pi G=2.79 \pm 0.04 M_{\odot}$. Our value is consistent at the $1 \sigma$ level with previous results by Marsh et al. $(1994, f(M)=2.71 \pm 0.06)$ and Neilsen et al. $(2008, f(M)=$ $2.76 \pm 0.01)$.

The derived radial velocity of the centre of mass of the system also agrees at the $1 \sigma$ with previous studies (e.g. $\gamma=4 \pm$ $2 \mathrm{~km} \mathrm{~s}^{-1}$, Marsh et al. 1994). Our high-resolution data has better spectral resolution than all previous data by a factor greater than 10. For instance, the studies presented by Marsh et al. (1994) and Neilsen et al. (2008) used spectra with a resolving power of 70 and $130 \mathrm{~km} \mathrm{~s}^{-1}$, respectively. Despite this, Neilsen et al. (2008) provides a significantly lower uncertainty for the semiamplitude velocity of $0.5 \mathrm{~km} \mathrm{~s}^{-1}$ given their resolving power. This is because our orbital coverage is much less complete than previous studies, especially around orbital phase 0.75 .

\subsection{Rotational velocity}

Following Marsh et al. (1994), we also computed the optimal $v \sin i$ by subtracting broadened versions of the template star, in steps of $1 \mathrm{~km} \mathrm{~s}^{-1}$, and minimizing the residual. We used a spherical rotational profile with linearized limb darkening $\epsilon=0.81$, which is appropriate for the stellar parameters of the donor star (González Hernández et al. 2004) and our wavelength range $\sim 5250 \AA$ (Al-Naimiy 1978). The best fit corresponds to a $v \sin i=96 \pm 0.8 \mathrm{~km} \mathrm{~s}^{-1}$, but the error is purely formal since it does not account for systematics due to our choice of $\epsilon$, which is adequate for the continuum but likely overestimated for the absorption lines (Collins \& Truax 1995). In an attempt to derive a more realistic error, we also computed the rotational broadening 


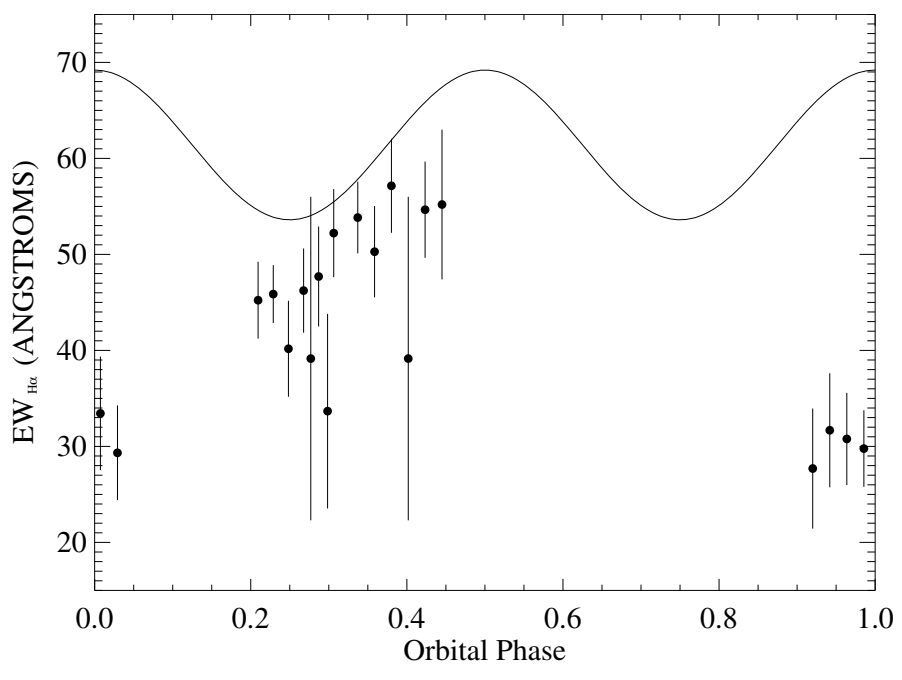

Fig. 2. Equivalent width of $\mathrm{H} \alpha$ profiles of the 20 UVES spectra of A0620-00 versus orbital phase of the secondary star. The solid line is the solution of Marsh et al. (1994) for comparison.

for the extreme case $\epsilon=0$ and find $v \sin i=87 \pm 0.7 \mathrm{~km} \mathrm{~s}^{-1}$. A more conservative value is then provided by the mean of the two determinations, i.e., $v \sin i=92 \pm 5 \mathrm{~km} \mathrm{~s}^{-1}$. As a result of the optimal subtraction we also find that the donor star contributes $\sim 80 \%$ to the total light in the $\mathrm{H} \beta$ region and $\sim 85 \%$ in the $\mathrm{H} \alpha$ region. At this point we note that the absorption features of the secondary star can be smeared by as much as $53 \mathrm{~km} \mathrm{~s}^{-1}$ according to the length of the exposure times and the orbital phase of the observations. We also tried to correct for this by simulating the smearing in the template spectrum according to Casares et al. (1997) but found that the effect is completely negligible.

In the case of tidally locked Roche-lobe filling stars, the rotational velocity relates to the velocity semiamplitude, $K_{2}$, and the mass ratio, $q=M_{2} / M_{1}=K_{1} / K_{2}$, through the expression $v \sin i \simeq 0.462 K_{2} q^{1 / 3}(1+q)^{2 / 3}$ (e.g. Wade \& Horne 1988). Our derived rotational velocity, combined with our value of $K_{2}$, implies a binary mass ratio $q=M_{2} / M_{1}=0.081 \pm 0.010$. However, because of the Roche lobe geometry, $v \sin i$ displays a $\sim 10 \%$ modulation with maxima at the orbital quadratures (Casares et al. 1996). Most of our spectra are located at orbital phases around 0.25 , so our previous determination is likely to be overestimated by a factor $\sim 5 \%$, which would make it consistent with Marsh et al. (1994) and Neilsen et al. (2008). Therefore, we think that the value provided by Marsh et al. (1994), $v \sin i=83 \pm 5$, is more realistic. Using this result and our value for $K_{2}$, we obtain $q=0.062 \pm 0.010$, which is the same value given in Neilsen et al. (2008). This, combined with the mass function, provides a minimum mass for the compact object of $M_{1} \sin ^{3} i=f(M) *(1+q)^{2}=$ $3.15 \pm 0.10 M_{\odot}$.

\section{Ellipsoidal variations in the $\mathrm{H} \alpha$ line}

We determined the equivalent width $(E W)$ of $\mathrm{H} \alpha$ profiles for the 20 UVES spectra of A0620-00 by adding numerically the normalized fluxes multiplied by the wavelength step. In Fig. 2 we depict the evolution of the $E W$ of $\mathrm{H} \alpha$ with orbital phase. The error bars were determined by measuring the change in $E W$ from varying the continuum location according to the $S / N$ in the continuum regions. Despite our limited phase coverage, the orbital variation in the $E W \mathrm{~s}$ hints at the ellipsoidal varibility seen by
Marsh et al. (1994) and Neilsen et al. (2008) due to the dominance of the donor star's light.

We have also displayed for comparison the solution of the fit to a sinusoid plus a constant to the data of Marsh et al. (1994), $E W(\AA)=61.4+7.8 \cos 4 \pi \phi$. Our mean $E W$ is $43 \AA$, significantly lower than in Marsh et al. (1994), whose spectroscopic data were obtained in December 1991, and much lower than that in Neilsen et al. (2008), whose data were obtained in December 2006. This may come from an increase in the continuum from the accretion disk, a decrease in the $\mathrm{H} \alpha$ flux or a combination of the two. Despite being in quiescence, A0620-00 shows significant variability and different states associated with changes in disk activity (e.g. Cantrell et al. 2008). Therefore, the lower $E W$ seen in our spectra over previous studies could well be associated to a different level of accretion disk activity. The relative contribution of the accretion disk to the continuum in the $\mathrm{H} \alpha$ region is $\sim 15 \%$ in our data and $\sim 6 \%$ in Marsh et al. (1994). This, combined with the variation in $E W \mathrm{~s}$ between the two data sets, implies that the $\mathrm{H} \alpha$ flux has dropped $\sim 33 \%$ in our data with respect to Marsh et al. (1994). In fact, Cantrell et al. (2008) find that A0620-00 was in a lower level of activity (what they called "passive" state) most of the time between December 1999 and December 2003. Our observations were taken in December 2000 , when the system was in the "passive" state, and this seems to be the reason for the low level of $\mathrm{H} \alpha$ flux that we see.

\section{Doppler images of $\mathrm{H} \alpha$ and $\mathrm{H} \beta$}

We have used Doppler tomography (Marsh \& Horne 1988) to map the $\mathrm{H} \alpha$ and $\mathrm{H} \beta$ emission in A0620-00. By combining the orbitally-resolved line profiles we were able to reconstruct the brightness distribution of the system in velocity space. The result is displayed in Fig. 3. The Doppler maps were built by combining the 20 profiles. The spectra of $\mathrm{H} \alpha$ and $\mathrm{H} \beta$ were continuum subtracted, leaving the pure line emission, and rebinned to a velocity scale of $9 \mathrm{~km} \mathrm{~s}^{-1}$ per pixel. The top panels show trailed spectra and the bottom panels the corresponding Doppler images. The location of the main components in the system, such as the Roche lobe of the secondary star and the predicted gas stream trajectory and the Keplerian trajectory of the disk along the stream are indicated. These tracks were calculated by adopting the values $K_{2} \sim 437 \mathrm{~km} \mathrm{~s}^{-1}$ and $q=M_{2} / M_{1} \sim 0.06$. Although the disk activity seems to be lower than in previous studies (see Sect. 3), we can see a clear detection of the bright spot between the velocity trajectories of the gas stream and of the disk along the stream, as previously reported in Marsh et al. (1994); Neilsen et al. (2008) and in other systems.

The Doppler maps shown in Fig. 3 were constructed without subtracting any K-type template spectrum from the original data. We note here an intense $\mathrm{H} \alpha$ emission exactly at the position of the secondary star. The emission coming from the secondary star can also be identified through the short tracks of the expected Swave emission on the trailed spectrogram. The $\mathrm{H} \beta$ Doppler map also shows emission around the position of the Roche lobe but it is less clear. The $S / N$ of the spectra at $\mathrm{H} \beta$ is significantly lower and the line is also close the edge of the CCD, which makes this map somewhat uncertain.

\section{Narrow $\mathrm{H} \alpha$ component emission from the secondary star}

Casares et al. (1997) have already found a narrow $\mathrm{H} \alpha$ emission line associated to the donor star in the spectra of the black hole 


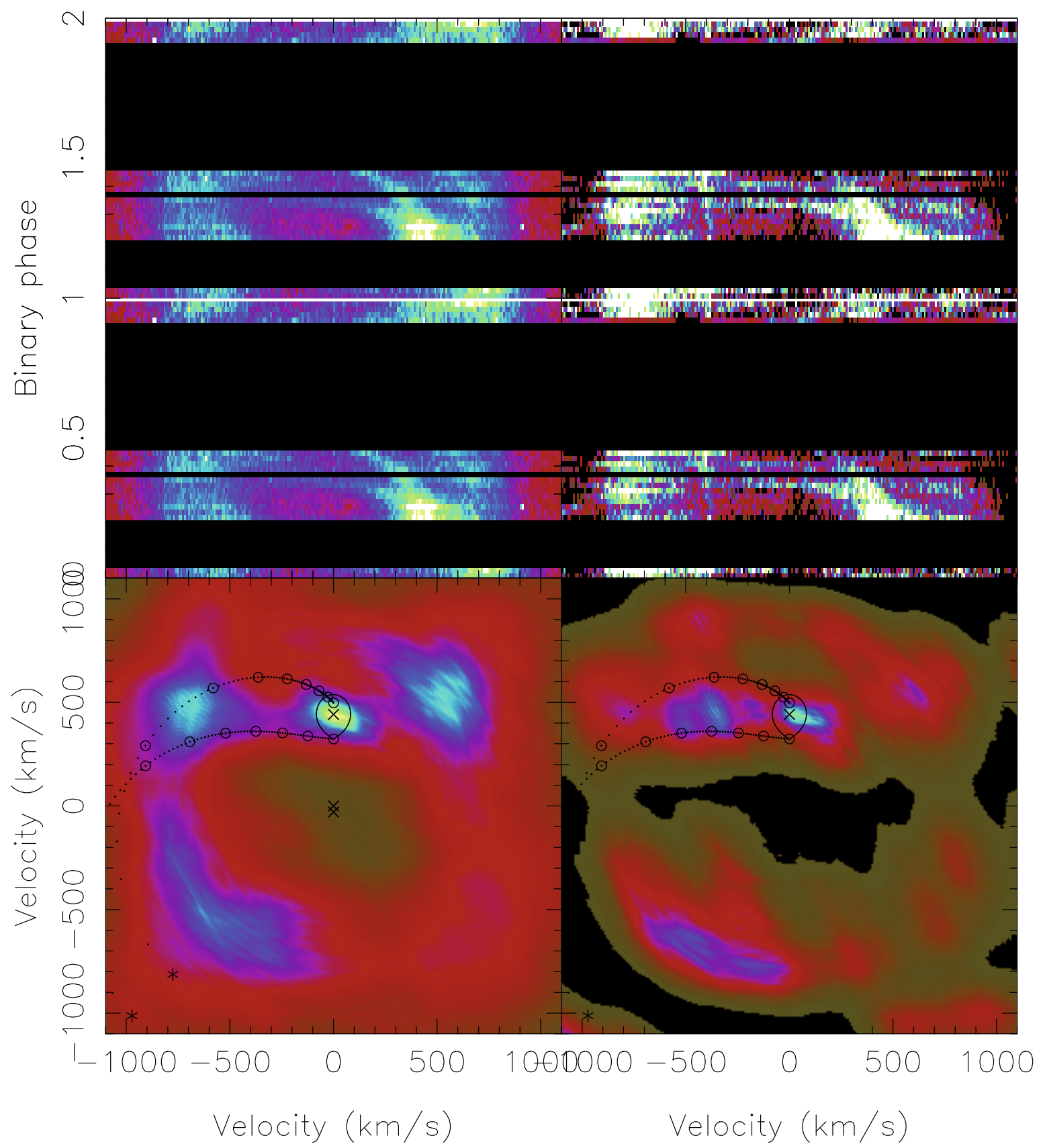

Fig. 3. Doppler images of $\mathrm{H} \alpha$ and $\mathrm{H} \beta$ for the UVES spectroscopic data. The trailed spectra are displayed in the top panels where several gaps in phase appear because the observations were taken over three different nights. Doppler images are depicted in the bottom panels. The predicted velocities of the gas stream (which starts from the inner Lagrangian point) and of the disk along the gas stream are plotted for $K_{2}=437.1 \mathrm{~km} \mathrm{~s} \mathrm{~s}^{-1}$ and $q=M_{2} / M_{1}=0.062$. Asterisks mark turning points in distance from the compact object. Circles have been plotted every $0.1 R_{L 1}$, and dots every $0.01 R_{L 1}$ along the streams.

binary Nova Muscae 1991. This line was tentatively associated to chromospheric activity of the secondary star in this system. It is known that rapidly rotating stars such as young T-Tauri stars (e.g. Appenzeller \& Mundt 1989) and/or old, non-interacting RS CVn binaries (e.g. Herbig 1985; Fekel et al. 1986), show a high level of chromospheric activity, with significant filling-in of the $\mathrm{H} \alpha$ core and, occasionally, reversal into emission. Rapidly rotating and convective stars are capable of manifesting surface magnetic fields through the dynamo process (Parker 1955). The induced magnetic fields lead to confinement and heating of plasma, which produce chromospheric and coronal emissions (e.g Ca II $\mathrm{H}$ and $\mathrm{K}$ lines, $\mathrm{H} \alpha$ and $\mathrm{X}$-rays). Observations of these phenomena in solar type stars have shown that the chromospheric activity increases with the rotation rate (e.g. Noyes et al. 1984).

Companion stars of X-ray transients are tidally locked, with short orbital periods, hence high rotational velocities. Thus, it is not surprinsing that the secondary star in A0620-00, with $v \sin i \sim 83 \mathrm{~km} \mathrm{~s}^{-1}$, is chromospherically active. However, this is the first time that chromospheric emission from the secondary 


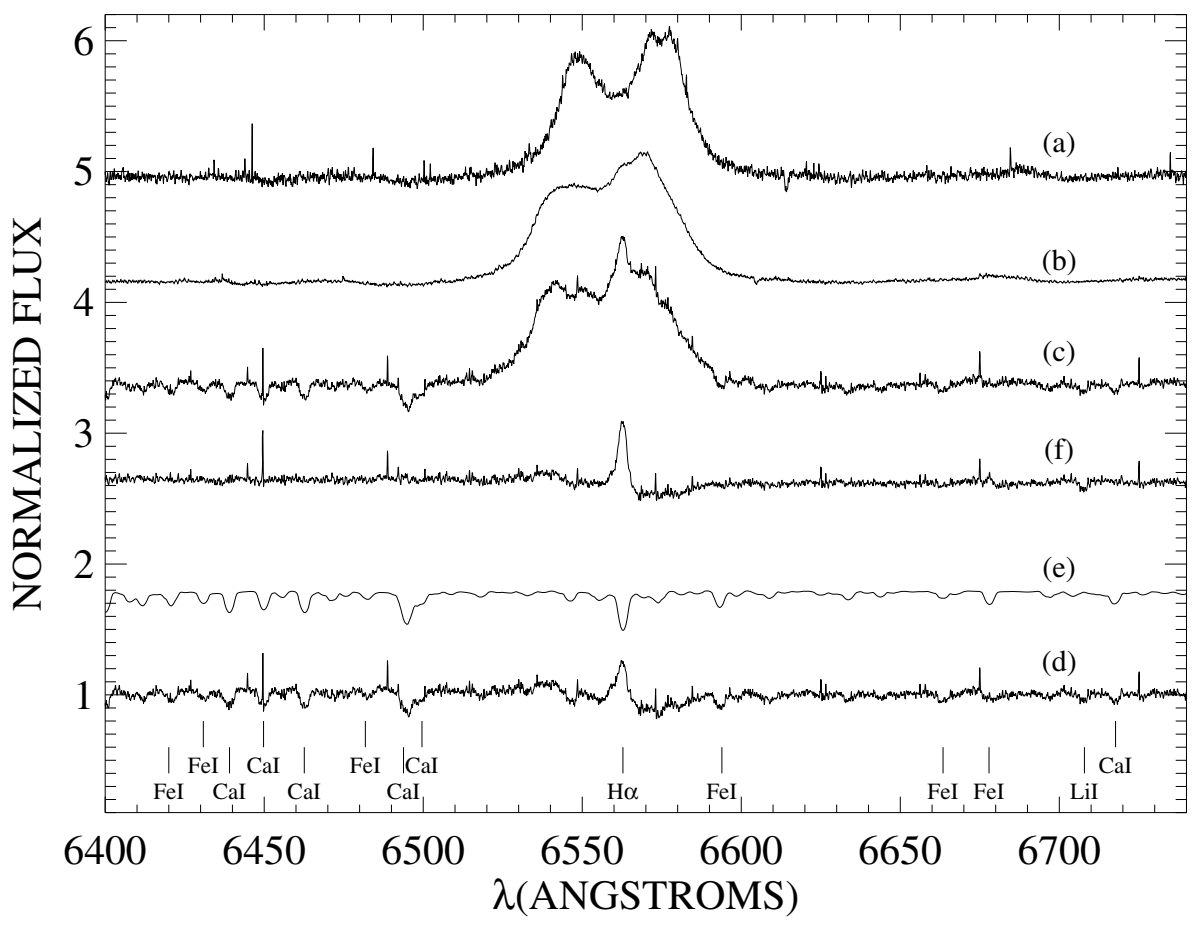

Fig. 4. Isolating the $\mathrm{H} \alpha$ narrow component in emission coming from the secondary star in A0620-00 from the H $\alpha$ profiles of the accretion disk. The spectra displayed are, from top to bottom; a) average $\mathrm{H} \alpha$ profile in the rest frame of the system centre-of-mass; b) Doppler-corrected average of the spectrum (1) in the rest frame of the secondary star; c) Doppler corrected average spectrum of the secondary star; d)=c) - b) spectrum of the secondary star without emission lines from the accretion disk, showing the narrow emission $\mathrm{H} \alpha$ component of the secondary star; e) spectrum of the K-type template star properly broadened; and $\mathbf{f})=\mathbf{d}$ ) $-0.76 \mathbf{e}$ ) is chromospheric spectrum of the secondary star. Arbitrary offsets have been applied to the spectra for the sake of clarity.

star has been clearly detected in this system before subtracting the spectrum of a template K-type star. Marsh et al. (1994) do detect a narrow $\mathrm{S}$-wave in their trailed spectra but only after subtracting the template star, which, as a matter of fact, provides a limit on the chromospheric emission set by the equivalent width of the $\mathrm{H} \alpha$ absorption in the template star. The reason this chromospheric component has not been detected in previous studies seems to hold on variability arguments, perhaps related to the low level of accretion disk activity seen in our data (which otherwise might somehow dilute the chromospheric component) or simply caused by variations in the donor star's activity (magnetic cycles).

We attempted to detrend the narrow $\mathrm{H} \alpha$ component from the broad underlying accretion disk contribution. The K3-4.5V template spectrum used in this work shows an $\mathrm{H} \alpha$ line in absorption of $\sim 1.3-1.4 \AA$. This could be considered as a lower limit to the $E W$ of the chromospheric emission of the secondary star in A0620-00. In fact, the derived effective temperature of $T_{\text {eff }} \sim 4900 \mathrm{~K}$ by González Hernández et al. (2004) favours a slightly earlier spectral type, i.e. K2V, which would shift this limit up to $1.4-1.5 \AA$.

In the Doppler-corrected average of the 20 spectra of the system, this narrow component appears as a narrow feature on top of the broad doubled-peak $\mathrm{H} \alpha$ emission produced by the accretion disk (see spectrum (c) of Fig. 4). The emission of the accretion disk can be approximated by a Doppler-shifted average (in the rest frame of system centre-of-mass) of the double-peaked profile (spectrum (a) of Fig. 4). This is not exactly the real accretion disk emission profile since it also contains the smeared S-wave component of the secondary star. We then shifted in velocity 20 versions of spectrum (a) to the rest frame of the secondary star at the given phase of each individual spectrum and again computed the average using the same weights as before. The resulting profile (spectrum (b) of Fig. 4) was subsequently subtracted from the original Doppler-corrected average, i.e., spectrum (c), leaving the narrow $\mathrm{H} \alpha$ component associated with the secondary star completely isolated (spectrum (d) of Fig. 4). After this subtraction, we added a constant value equal to 1 since the continuum level of the resulting spectrum was at zero, in order to avoid to work with negative spectral points. We performed a second iteration of the whole process by first subtracting each original spectrum by spectrum (d), properly corrected by the star's velocity at each orbital phase, in order to eliminate the smeared narrow component of the secondary star from the profiles (a) and (b). In fact, the spectra displayed in Fig. 4 shows the results of this last iteration.

The spectrum (d) of Fig. 4 is the normalized spectrum of the secondary star without the accretion disk contribution. We measured the equivalent width of the narrow $\mathrm{H} \alpha$ component from the secondary star in this spectrum to be $1.40 \pm 0.29 \AA$. The error bar has been estimated by changing the position of the continuum, taking the $S / N$ of the spectrum of the secondary star into account. In order to measure the real chromospheric emission $\mathrm{H} \alpha$ line, one needs to subtract the photospheric component using a template star with the same spectral type (see e.g. Montes et al. 1995). We then subtracted a K-type template star properly broadened (spectrum (e)) and scaled by a veiling factor of 0.76 , and find an $E W$ of $2.15 \pm 0.26 \AA$ (spectrum (f)), which has to be corrected for the same veiling factor, leading to a final value of $E W(\mathrm{H} \alpha)=2.82 \pm 0.34 \AA$.

\section{Discussion}

Strassmeier et al. (1990) measured the $\mathrm{H} \alpha E W$ s of a sample of F6-M2 single and binary stars, showing that the $\mathrm{H} \alpha E W$ 
A\&A 516, A58 (2010)

Table 1. H $\alpha$ fluxes, equivalent widths, and Rossby numbers of the LMXBs displayed in Fig. 5.

\begin{tabular}{|c|c|c|c|c|c|c|c|c|c|}
\hline Object & $\begin{array}{r}E W(\mathrm{H} \alpha)^{a} \\
{[\AA]}\end{array}$ & $\begin{array}{c}T_{\text {eff }} \\
{[\mathrm{K}]}\end{array}$ & $\begin{array}{l}\log g \\
{[\operatorname{dex}]}\end{array}$ & $\begin{array}{r}\log F(\mathrm{H} \alpha) \\
{\left[10^{6} \mathrm{erg} \mathrm{cm}^{-2} \mathrm{~s}^{-1}\right]}\end{array}$ & $\begin{array}{r}\log R(\mathrm{H} \alpha) \\
{[\mathrm{dex}]}\end{array}$ & $\begin{array}{r}\tau_{\mathrm{c}}{ }^{b} \\
\text { [days] }\end{array}$ & $\begin{array}{r}P_{\text {orb }} \\
\text { [days] }\end{array}$ & $\begin{array}{r}\log R_{0} \\
{[\operatorname{dex}]}\end{array}$ & Refs. $^{c}$ \\
\hline A0620-00 & $2.82 \pm 0.34$ & $4900 \pm 100$ & 4.2 & $6.95 \pm 0.05$ & $-3.57 \pm 0.06$ & 22.7 & 0.32 & -1.85 & 1,2 \\
\hline Nova Muscae 1991 & $3.65 \pm 0.32$ & $4500 \pm 100^{d}$ & $4.1^{d}$ & $6.89 \pm 0.08$ & $-3.48 \pm 0.09$ & 24.6 & 0.43 & -1.74 & 3,4 \\
\hline Cen $\mathrm{X}-4^{e}$ & $2.27 \pm 0.67$ & $4500 \pm 100$ & 3.9 & $6.68 \pm 0.11$ & $-3.68 \pm 0.12$ & 24.7 & 0.63 & -1.58 & 5,6 \\
\hline Cen $X-4^{f}$ & $4.40 \pm 0.50$ & $4500 \pm 100$ & 3.9 & $6.96 \pm 0.05$ & $-3.40 \pm 0.06$ & 24.7 & 0.63 & -1.58 & 6,7 \\
\hline Nova Scorpii 1994 & $10.0 \pm 0.50$ & $6100 \pm 100$ & 3.7 & $7.92 \pm 0.02$ & $-2.97 \pm 0.04$ & 7.6 & 2.62 & -0.60 & $8,9,10$ \\
\hline
\end{tabular}

Notes. ${ }^{a}$ Corrected equivalent width of narrow $\mathrm{H} \alpha$ component of the secondary star. ${ }^{b}$ Convective turnover time derived from the equations in Noyes et al. (1984) for dwarfs stars and using the turnover times given in Basri (1987) for subgiant and giant stars. ${ }^{c}$ References: (1) González Hernández et al. (2004), (2) McClintock \& Remillard (1986), (3) Casares et al. (1997), (4) Remillard et al. (1992), (5) Torres et al. (2002), (6) González Hernández et al. (2005), (7) D’Avanzo et al. (2005), (8) Shahbaz et al. (1999), (9) González Hernández et al. (2008a), (10) van der Hooft et al. (1998). ${ }^{d}$ Adopted values according to the effective temperature and surface gravity for the spectral type and orbital period. ${ }^{e} \mathrm{H} \alpha$ equivalent width obtained from Torres et al. (2002). ${ }^{f} \mathrm{H} \alpha$ equivalent width obtained from D’Avanzo et al. (2005).

increases towards shorter rotation periods, $P$. Extrapolation of this trend $E W$ vs. $P$ at the orbital period of A0620-00 provides an expected $E W$ of $\sim 2.3 \AA$, assuming that the secondary star is a dwarf main-sequence star and that its rotation is sychronized with the orbital motion. This $E W$ is marginally consistent (at $1.5 \sigma$ ) with the observed $E W$ of the secondary star. However, the extrapolation might not be adequate since chromospheric features powered by rotation typically saturates for period shorter than 1-3 days (e.g. Cardini \& Cassatella 2007, for chromospheric Mg II lines).

We can also compare the $\mathrm{H} \alpha E W$, converted into flux following the approach of Soderblom et al. (1993), with the observations of field stars and binaries. Thus, the $\mathrm{H} \alpha$ flux at the stellar surface, is $F_{\mathrm{H} \alpha}=8.8 \pm 1.1 \times 10^{6} \mathrm{erg} \mathrm{cm}^{-2} \mathrm{~s}^{-1}$ (see Table 1). We use a similar prescription than that of Eq. (2) in Soderblom et al. (1993), i.e. $F_{\mathrm{H} \alpha}=\mathrm{EW}(\mathrm{H} \alpha) F_{\mathrm{c}}$, where $F_{\mathrm{c}}$ is the continuum flux at $\mathrm{H} \alpha$ and is derived using the flux calibration of Hall (1996), $\log F_{\mathrm{c}}=7.538-1.081(B-V)_{0}$. Here we used $(B-V)_{0}=0.965$ estimated from theoretical colours (Bessell et al. 1998) according to the stellar parameters of the secondary star (González Hernández et al. 2004). The surface flux is usually normalized to the bolometric flux, i.e. $R_{\mathrm{H} \alpha}=F_{\mathrm{H} \alpha} / \sigma T_{\mathrm{eff}}^{4}$. The connection between chromospheric activity and rotation is obtained by studying the correlation $R_{\mathrm{H} \alpha}$ with the Rossby number $R_{0}=P / \tau_{\mathrm{c}} \lesssim 2 \pi R_{\star} / \tau_{\mathrm{c}} v \sin i$, where $\tau_{\mathrm{c}}$ is the convective turnover time, $P$, the rotation period of the secondary star and $R_{\star}$ its radius. Using the Rossby number is usually preferred over $P$ and $v \sin i$ since it does not depend on the mass of the star.

For the case of A0620-00, we adopt $T_{\text {eff }}=4900 \mathrm{~K}$ (González Hernández et al. 2004), $P=P_{\text {orb }}=0.32 \mathrm{~d}$ and $\tau_{\mathrm{c}} \sim 23 \mathrm{~d}$, computed from Eq. (4) of Noyes et al. (1984). We derive $\log R_{\mathrm{H} \alpha}=-3.57 \pm 0.06$ and $\log R_{0}=-1.85$, and this is listed in Table 1, together with values derived for other quiescent $\mathrm{X}$-ray transients. In Fig. 5 we compare these values with those of chromospherically active single stars (López-Santiago et al. 2010) and binary systems (RS CVn and BY Dra classes) from Montes et al. (1995). Our value of the Rossby number places the secondary star of A0620-00 in the region of activity saturation, where all measurements tend to the same average value of $\log R_{\mathrm{H} \alpha} \sim-3.7$, and is consistent with the general trend. The other X-ray binaries show similar results, except for the black hole X-ray binary Nova Scorpii 1994, which displays too high an $R_{\mathrm{H} \alpha}$ value for its relatively low Rossby number. This is even more evident when comparing the $\mathrm{H} \alpha$ fluxes, with $\log F_{\mathrm{H} \alpha} \sim 7.9$ for Nova Scorpii 1994, significantly higher, by almost one order

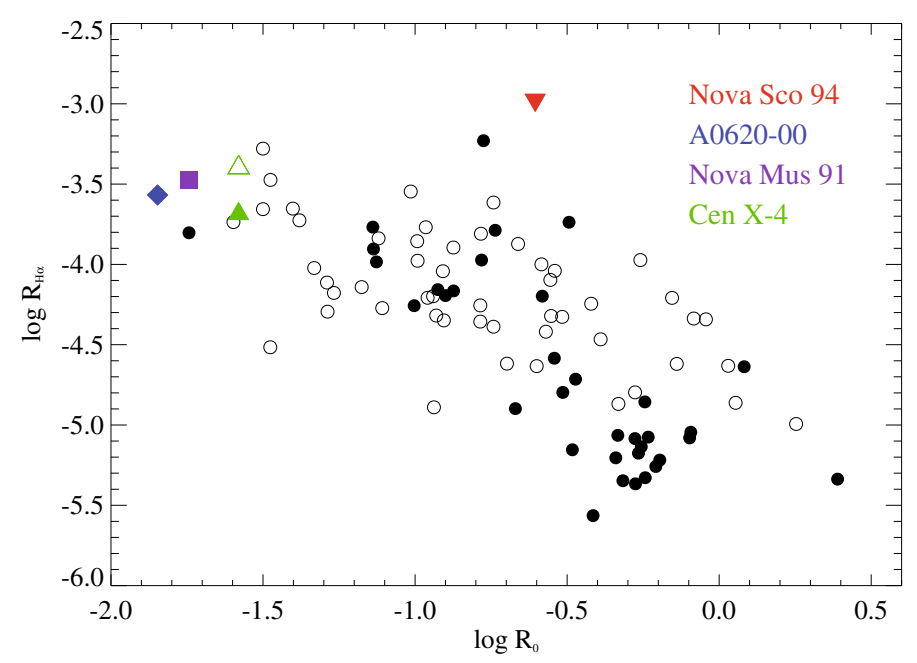

Fig. 5. Chromospheric flux ratios vs. Rossby numbers of chromospherically active single stars from López-Santiago et al. (2010, filled circles) and chromospherically active binary systems (RS CVn and BY Dra classes) from Montes et al. (1995, open circles). Low-mass X-ray binaries are also depicted: A0620-00 (diamond), Nova Muscae 1991 (square), Cen X-4 (filled triangle, from Torres et al. 2002; open triangle, from D'avanzo et al. 2005), and Nova Scorpii 1994 (inverted triangle).

of magnitude, than the saturation level at $\log F_{\mathrm{H} \alpha} \sim 6.9$. In addition, D'Avanzo et al. (2005) also suggest that the $\mathrm{H} \alpha E W$ of the secondary star in Cen X-4 is correlated with the veiling of the accretion disk (see Table 1), by comparing their values with those given by Torres et al. (2002). Although this is not expected in a chromospheric activiy scenario, the two Cen X-4 points in Fig. 5 fall in the saturation region, together with other X-ray and chromospherically active binaries. The behaviour seen in Nova Scorpii 1994 and Cen X-4 suggests that rapid rotation might not be the only explanation for the narrow $\mathrm{H} \alpha$ feature, at least in these LMXBs, but perhaps a combination of rotation and reprocessing of X-ray flux from the accretion disk into $\mathrm{H} \alpha$ photons in the secondary star. It is therefore worth investigating in A0620-00 if X-ray heating could be an alternative explanation for this feature.

The system A0620-00 has been observed in quiescence with the Chandra X-ray satellite, providing a $0.5-10 \mathrm{keV}$ unabsorbed flux $F_{\mathrm{X}, 0}=6.7_{-2.3}^{+0.8} \times 10^{-14} \mathrm{erg} \mathrm{cm}^{-2} \mathrm{~s}^{-1}$ (Gallo et al. 2006). The X-ray flux at the stellar surface can be computed as $F_{\mathrm{X}, \star}=F_{\mathrm{X}, 0}(d / a)^{2}=9.5 \times 10^{6} \mathrm{erg} \mathrm{cm}^{-2} \mathrm{~s}^{-1}$, where we 
have adopted an orbital separation $a=4.47 R_{\odot}$ and a distance $d=1.2 \mathrm{kpc}$. This means that almost $92 \%$ of the incident X-ray radiation would have to be reprocessed to $\mathrm{H} \alpha$ photons in order to power the observed $\mathrm{H} \alpha$ emission. Following Hynes et al. (2002), $F_{\mathrm{H} \alpha, \star}=f_{1} f_{2} F_{\mathrm{X}, \star}$, where $f_{1}$ is the fraction of X-ray emission intercepted by the companion; i.e., the solid angle subtended by the companion from the compact object $\left(f_{1}=\left[R_{\star} /(2 a)\right]^{2}\right)$, and $f_{2} \lesssim 0.3$ is the fraction of input energy emitted in $\mathrm{H} \alpha$ (Hynes et al. 2002, and references therein). Adopting $R_{\star}=1.1 R_{\odot}$ from González Hernández et al. (2004), we obtain $F_{\mathrm{H} \alpha, \star} \lesssim 4.5 \times 10^{-3} F_{\mathrm{X}, \star}$. This number is significantly lower than the observed value, which indicates that the incident $\mathrm{X}$-ray irradiation is not enough to produce the narrow $\mathrm{H} \alpha$ line in the secondary star. D'Avanzo et al. (2005) have also derived these quantities for the case of the neutron star binary Cen X-4 and find both estimates to be consistent, because the X-ray flux in Cen X-4 is $F_{\mathrm{X}, \mathrm{CenX}-4}=5 \times 10^{8} \mathrm{erg} \mathrm{cm}^{-2} \mathrm{~s}^{-1}$, i.e. almost two orders of magnitude higher than in A0620-00.

There is still a remote posibility that the source of irradiating photons is hidden away in the EUV (extreme UV) energy range, between 100-1200 $\AA$. Although this energy range is not directly observed, we can guess roughly how much flux is involved through interpolating the nearby soft X-ray and the farUV (FUV) emission. The FUV flux (in the range 1350-2200 $⿱$ ) has been determined at $F_{\mathrm{FUV}, 0}=0.2-1.4 \times 10^{-13} \mathrm{erg} \mathrm{cm}^{-2} \mathrm{~s}^{-1}$ (McClintock et al. 1995), i.e. similar to the X-ray flux. This and the absence of He II $\lambda 4686 \AA$ line emission in the optical spectrum (Marsh et al. 1994) suggest that flux in the

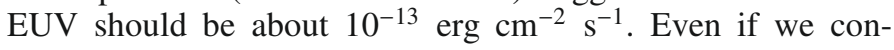
sider all the ionizing photons (X-ray+EUV+FUV), the total flux would be roughly three times the X-ray flux, i.e. $\sim 2 \times$ $10^{-13} \mathrm{erg} \mathrm{cm}^{-2} \mathrm{~s}^{-1}$. Therefore, if we assume that the incident irradiation is $F_{\mathrm{X}-\mathrm{UV}, \star}=3 F_{\mathrm{X}, \star}$, then the $31 \%$ of the incident radiation would have to be reprocessed to $\mathrm{H} \alpha$ photons in order to power the observed $\mathrm{H} \alpha$ emission, which still is too high a fraction compared to the fraction previously estimated as below $1 \%$.

\section{Summary}

We have presented high-resolution UVES/VLT spectroscopy of the black hole binary A0620-00 at quiescence. Our orbital parameters are consistent with previous studies by Marsh et al. (1994) and Neilsen et al. (2008). In particular, we derived $P_{\text {orb }}=$ $0.32301405(1) \mathrm{d}$ and $K_{2}=437.1 \pm 2.0 \mathrm{~km} \mathrm{~s}^{-1}$. These values, together with the mass ratio $q=M_{2} / M_{1}=0.062 \pm 0.010$, imply a minimum mass for the compact object of $M_{1} \sin ^{3} i=$ $3.15 \pm 0.10 M_{\odot}$.

We also performed Doppler tomography of the accretion disk emission and discovered emission at the position of the secondary star in the Doppler maps of $\mathrm{H} \alpha$ and $\mathrm{H} \beta$, which were not detected in previous studies. We isolated the chromospheric $\mathrm{H} \alpha$ emission from the secondary star and measured an equivalent width of $2.82 \pm 0.34 \AA$. This equivalent width is too large to be explained by X-ray and/or UV irradiation from the inner accretion disk; therefore, chromospheric activity, induced by rapid rotation, seems the most likely origin of this feature in the black hole binary A0620-00.

Acknowledgements. J.I.G.H. acknowledges support from the project AYA200800695 of the Spanish Ministry of Education and Science. J.C. acknowledges support from the Spanish Ministry of Science and Technology through the project AYA2007-66887. This work has been partially funded by the Spanish MICINN under the Consolider-Ingenio 2010 Program grant CSD2006-00070: First Science with the GTC (http://www.iac.es/consolider-ingenio-gtc). We are grateful to Tom Marsh for the use of the MOLLY analysis package. J.I.G.H. is grateful to Javier López Santiago for providing us with the data of chromospherically active single and binary stars. J.I.G.H. acknowledges thanks helpful discussions with Javier López Santiago, David Montes and Raquel Martínez Arnáiz.

\section{References}

Al-Naimiy, H. M. 1978, Ap\&SS, 53, 181

Appenzeller, I., \& Mundt, R. 1989, A\&ARv, 1, 291

Basri, G. 1987, ApJ, 316, 377

Bessell, M. S., Castelli, F., \& Plez, B. 1998, A\&A, 333, 231

Cardini, D., \& Cassatella, A. 2007, ApJ, 666, 393

Cantrell, A. G., Bailyn, C. D., McClintock, J. E., \& Orosz, J. A. 2008, ApJ, 673, L159

Cantrell, A. G. Bailyn, C. D., Crosz, J. A., et al. 2010, ApJ, 710, 1127

Casares, J., Mouchet., M., Martínez-Pais, I. G., \& Harlaftis, E. T. 1996, MNRAS, 282,182

Casares, J., Martin, E. L., Charles, P. A., Molaro, P., \& Rebolo, R. 1997, New Astron., 1, 299

Collins, G. W. II, \& Truax, R. J. 1995, ApJ, 439, 860

D’Avanzo, P., Campana, S., Casares, J., et al. 2005, A\&A, 444, 905

Elvis, M., Page, C. G., Pounds, K. A., Ricketts, M. J., \& Turner, M. J. L. 1975, Nature, 257, 656

Fekel, F. C., Moffett, T. J., \& Henry, G. W. 1986, ApJS, 60, 551

Froning, C. S., Robinson, E. L., \& Bitner, M. A. 2007, ApJ, 663, 1215

Gallo, E., Fender, R. P., Miller-Jones, J. C. A., et al. 2006, MNRAS, 370, 1351

Gelino, D. M., Harrison, T. E., \& Orosz, J. A. 2001, AJ, 122, 2668

Gelino, D. M., Balman, Ş., Kililoğlu, Ü., et al. 2006, ApJ, 642, 438

González Hernández, J. I., Rebolo, R., Israelian, G., et al. 2004, ApJ, 609, 988

González Hernández, J. I., Rebolo, R., Israelian, G., et al. 2005, ApJ, 630, 495

González Hernández, J. I., Rebolo, R., Israelian, G., et al. 2006, ApJ, 644, L49

González Hernández, J. I., Rebolo, R., \& Israelian, G. 2008a, A\&A, 478, 203

González Hernández, J. I., Rebolo, R., Israelian, G., et al. 2008b, ApJ, 679, 732

Hall, J. C. 1996, PASP, 108, 313

Harrison, T. E., Howell, S. B., Szkody, P., \& Cordova, F. A. 2007, AJ, 133, 162

Harlaftis, E. T., Steeghs, D., Horne, K., \& Filippenko, A. V. 1997, AJ, 114, 1170

Herbig, G. H. 1985, ApJ, 289, 269

van der Hooft, F., Heemskerk, M. H. M., Alberts, F., \& van Paradijs, J. 1998, A\&A, 329, 538

Hynes, R. I., Zurita, C., Haswell, C. A., et al. 2002, MNRAS, 330, 1009

Hynes, R. I., Robinson, E. L., \& Bitner, M. 2005, ApJ, 630, 405

López-Santiago, J., Montes, D., Galvez-Ortiz, M. C., et al. 2010, A\&A, 514, A97

Marsh, T. R., \& Horne, K. 1988, MNRAS, 235, 269

Marsh, T. R., Robinson, E. L., \& Wood, J. H. 1994, MNRAS, 266, 137

McClintock, J. E., \& Remillard, R. A. 1986, ApJ, 308, 110

McClintock, J. E., Horne, K., \& Remillard, R. A. 1995, ApJ, 442, 358

Montes, D., Fernandez-Figueroa, M. J., de Castro, E., \& Cornide, M. 1995, A\&AS, 109, 135

Murdin, P., Allen, D. A., Morton, D. C., Whelan, J. A. J., \& Thomas, R. M. 1980, MNRAS, 192, 709

Neilsen, J., Steeghs, D., \& Vrtilek, S. D. 2008, MNRAS, 384, 849

Noyes, R. W., Hartmann, L. W., Baliunas, S. L., Duncan, D. K., \& Vaughan, A. H. 1984, ApJ, 279, 763

Oke, J. B. 1977, ApJ, 217, 181

Orosz, J. A., Bailyn, C. D., Remillard, R. A., McClintock, J. E., \& Foltz, C. B. 1994, ApJ, 436, 848

Parker, E. N. 1955, ApJ, 122, 293

Remillard, R. A., McClintock, J. E., \& Bailyn, C. D. 1992, ApJ, 399, L145

Shahbaz, T., van der Hooft, F., Casares, J., Charles, P. A., \& van Paradijs, J. 1999, MNRAS, 306, 89

Shahbaz, T., Hynes, R. I., Charles, P. A., et al. 2004, MNRAS, 354, 31

Soderblom, D. R., Stauffer, J. R., Hudon, J. D., \& Jones, B. F. 1993, ApJS, 85, 315

Strassmeier, K. G., Fekel, F. C., Bopp, B. W., Dempsey, R. C., \& Henry, G. W. 1990, ApJS, 72, 191

Torres, M. A. P., Casares, J., Martínez-Pais, I. G., \& Charles, P. A. 2002, MNRAS, 334, 233

Wade, R. A., \& Horne, K. 1988, ApJ, 324, 411 